\title{
BENCHMARKING DE INDICADORES DE QUALIDADE E DIMENSIONAMENTO DE PESSOAL DE ENFERMAGEM ENTRE UNIDADES HOSPITALARES
}

\author{
BENCHMARKING OF QUALITY INDICATORS AND \\ DIMENSIONING OF NURSING STAFF AMONG \\ HOSPITAL UNITS
}

\section{BENCHMARKING DE INDICADORES DE CALIDAD Y DIMENSIONAMIENTO DEL PERSONAL DE ENFERMERÍA ENTRE UNIDADES HOSPITALARIAS}

\author{
João Lucas Campos de Oliveira ${ }^{1}$ \\ Margani Cadore Weis Maia ${ }^{2}$ \\ Ana Maria Müller de Magalhães ${ }^{3}$ \\ Rúbia Marcela Rodrigues Moraes ${ }^{4}$ \\ Michelle Dornelles Santarem ${ }^{5}$ \\ Thamyres Laiz Oliveira Aquino ${ }^{6}$ \\ Samanta de Cassia da Silva ${ }^{7}$
}

\begin{abstract}
Como citar este artigo: Oliveira JLC, Maia MCW, Magalhães AMM, Moraes RMR, Santarem MD, Aquino TLO, et al. Benchmarking de indicadores de qualidade e dimensionamento de pessoal de enfermagem entre unidades hospitalares. Rev baiana enferm. 2020;34:e37756.
\end{abstract}

Objetivo: realizar benchmarking interno de indicadores de qualidade e do dimensionamento de pessoal de enfermagem entre unidades de internação hospitalar. Método: estudo transversal. Foram levantados/avaliados nove indicadores de qualidade por sítios de observação nas unidades de internação em clínica médica $(n=450)$ e cirúrgica (n=274) de hospital público do Centro-Oeste, Brasil, além da aplicação de Sistema de Classificação de Pacientes, para o dimensionamento de pessoal. Empregou-se análise estatística descritiva (no dimensionamento) e inferencial (para os indicadores). Resultados: houve diferença significativa (p-valor $<0,0001$ ) na conformidade da identificação do leito (melhor na unidade cirúrgica) e de acessos venosos (melhor na internação clínica). A classificação da qualidade foi equânime. A clínica médica apresentou déficit de enfermeiros (-11). Conclusão: a qualidade assistencial - mediada à métrica dos indicadores - foi equânime entre os setores de internação e o dimensionamento de pessoal foi discrepante, em virtude do evidente déficit de enfermeiros, dada à maior complexidade assistencial na clínica médica.

Descritores: Benchmarking. Indicadores de Qualidade em Assistência à Saúde. Dimensionamento. Gestão da Qualidade. Recursos Humanos de Enfermagem no Hospital. Pesquisa em Administração de Enfermagem.

\footnotetext{
Enfermeiro. Doutor em Enfermagem. Professor Adjunto da Universidade Federal do Rio Grande do Sul. Porto Alegre, Rio Grande do Sul, Brasil. joao-lucascampos@hotmail.com. https://orcid.org/0000-0002-1822-2360.

Enfermeira. Mestre em Enfermagem. Professora Assistente da Universidade Federal de Mato Grosso. Cuiabá, Mato Grosso, Brasil. https://orcid.org/0000-0002$5214-5960$.

Enfermeira. Doutora em Enfermagem. Professora Associada da Universidade Federal do Rio Grande do Sul. Porto Alegre, Rio Grande do Sul, Brasil. https://orcid. org/0000-0003-0691-7306.

4 Enfermeira. Enfermeira no Hospital Universitário Júlio Müller. Cuiabá, Mato Grosso, Brasil. https://orcid.org/0000-0003-|468-4| 5 |.

Enfermeira. Doutora em Epidemiologia. Professora Adjunta da Universidade Federal do Rio Grande do Sul. Porto Alegre, Rio Grande do Sul, Brasil. https://orcid. org/0000-0002-7046-7007.

Enfermeira. Pesquisadora independente. Cuiabá, Mato Grosso, Brasil. https://orcid.org/0000-0002-3616-2306.

Enfermeira. Pesquisadora independente. Cuiabá, Mato Grosso, Brasil. https://orcid.org/0000-0002-3443-9666.
} 
Objective: to perform internal benchmarking of quality indicators and the dimensioning of nursing staff between hospitalization units. Method: cross-sectional study. Nine quality indicators were collected/evaluated by observation sites in the medical $(n=450)$ and surgical $(n=274)$ bospital units of a public hospital in the Midwest, Brazil, in addition to the application of a Patient Classification System for the dimensioning of personnel. Descriptive statistical analysis (in sizing) and inferential (for indicators) were used. Results: there was a significant difference ( $p$-value $<0.0001$ ) in the compliance of bed identification (better in the surgical unit) and venous access (better in clinical hospitalization). The quality classification was equitable. The medical clinic presented a deficit of nurses (-11). Conclusion: the quality of care - mediated to the metrics of the indicators - was equitable between the hospitalization sectors and the personnel dimensioning was discrepant, due to the evident deficit of nurses, given the greater complexity of care in the medical clinic.

Descriptors: Benchmarking. Quality Indicators in Health Care. Sizing. Quality Management. Human Resources Nursing at the Hospital. Research in Nursing Administration.

Objetivo: realizar un benchmarking interno de los indicadores de calidad y dimensionamiento del personal de enfermería entre las unidades de hospitalización. Método: estudio transversal. Nueve indicadores de calidad fueron recogidos/evaluados por los sitios de observación en las unidades médicas (n-450) y quirúrgicas (n-274) de un hospital público en el Medio Oeste, Brasil, además de la aplicación de un Sistema de Clasificación de Pacientes para el dimensionamiento del personal. Se utilizaron análisis estadísticos descriptivos (en dimensionamiento) $e$ inferenciales (para indicadores). Resultados: hubo una diferencia significativa (valor p<0.0001) en el cumplimiento de la identificación de la cama (mejor en la unidad quirúrgica) y el acceso venoso (mejor en hospitalización clínica). La clasificación de calidad fue equitativa. La clinica médica presentaba un déficit de enfermeras (-11). Conclusión: la calidad de la atención - mediada a las métricas de los indicadores - era equitativa entre los sectores de hospitalización y el dimensionamiento del personal era discreto, debido al evidente déficit de enfermeras, dada la mayor complejidad de la atención en la clínica médica.

Descriptores: Benchmarking. Indicadores de Calidad en el Cuidado de la Salud. Tamaño. Gestión de la Calidad. Enfermería de Recursos Humanos en el Hospital. Investigación en Administración de Enfermería.

\section{Introdução}

O impulso por meios que viabilizem a qualidade na assistência à saúde e repercutam no cuidado qualificado e seguro tem sido o alvo na arena de discussões políticas e institucionais em todo o mundo, tanto por representar um direito do usuário como por ser um aspecto de interesse à sobrevivência e competividade organizacional $^{(1)}$. Neste escopo, a avaliação é uma ferramenta indispensável para a sustentabilidade produtiva. Uma forma de realizar a avaliação de desempenhos organizacionais é o benchmarking, que consiste em uma estratégia contínua e sistemática de comparação de produtos, serviços e processos de trabalho entre organizações reconhecidas como representantes de excelência, com a finalidade de melhoria contínua ${ }^{(2)}$.

Apesar de o benchmarking estar visivelmente atrelado à comparação de métricas entre competidores diretos, isto é, entre organizações distintas, uma possibilidade de seu emprego é por meio do benchmarking interno, no qual os resultados de indicadores são comparados entre setores/unidades/departamentos da mesma organização $^{(2)}$. O benchmarking interno desponta como uma possibilidade estratégica no setor saúde, já que a comparação entre instituições diferentes não é algo tão disseminado como em outros ramos de produção. Ademais, na saúde, a comparação de indicadores usualmente se dá em uma abordagem de tempo limitado, conforme atesta pesquisa de origem francesa ${ }^{(3)}$.

Mesmo que seja inegável o reconhecimento da necessidade de incrementar a melhoria da qualidade e segurança do paciente, e também do aumento aparentemente exponencial de estratégias que favorecem os processos de melhoria contínua - como o benchmarking - ainda existem diversos fatores que contribuem para deficiências no cuidado que impactam diretamente nesses bens, que incluem participações importantes da equipe de enfermagem ${ }^{(4-5)}$.

Pesquisas apontam que há um crescimento evidente na elevação da carga de trabalho da equipe de enfermagem e resultados negativos 
diretos na qualidade da assistência e na segurança do paciente. Sobre isso, resultados negativos de indicadores, como a média de permanência hospitalar, infecção urinária relacionada a procedimento invasivo, satisfação de pacientes com o cuidado de enfermagem, maior ocupação de leitos, ambiente de trabalho da equipe, quedas, e até mesmo mortalidade já foram relacionados ao aumento da carga de trabalho da enfermagem em estudos desenvolvidos em diversos países, como Coreia do $\mathrm{Sul}^{(4)}$, $\mathrm{Brasil}^{\left({ }^{(5)}\right.}$, Finlândia ${ }^{(6)}$ Taiwan $^{(7)}$ e Chile ${ }^{(8)}$.

Uma forma de racionalizar a carga de trabalho de enfermagem dá-se pelo provimento adequado de recursos humanos, vislumbrado, inclusive, pelo dimensionamento de pessoal ${ }^{(-10)}$. Este é entendido como um meio sistemático de previsão da quantidade de trabalhadores ajustada por categoria profissional, requerida para suprir as necessidades de assistência da enfermagem, direta ou indiretamente prestada à clientela, objetivando qualidade e segurança na assistência aos pacientes e segurança para os trabalhadores $^{(9-12)}$. No Brasil, os parâmetros para o dimensionamento de pessoal são estabelecidos pela Resolução do Conselho Federal de Enfermagem (COFEN) no 543/2017 $7^{(10)}$. Por sua vez, a qualidade em saúde/enfermagem é usualmente apreciada pela métrica de indicadores ${ }^{(2)}$.

No dimensionamento de pessoal de enfermagem, devem ser utilizadas metodologias e critérios específicos à realidade assistencial que permitam a determinação dos recursos humanos imprescindíveis às reais necessidades de cuidado $^{(9-12)}$. O Sistema de Classificação de Pacientes (SCP) caracteriza-se como ferramenta que permite classificar a clientela de acordo com o grau de dependência/nível de complexidade em relação ao cuidado requerido pela equipe de enfermagem. Ainda, pode viabilizar a determinação de horas necessárias para a prestação da assistência no ambiente hospitalar, variável elementar do dimensionamento de pessoal ${ }^{(12)}$.

Em hospitais, cabe ao enfermeiro o registro diário da classificação dos pacientes segundo o SCP, para subsidiar a composição do quadro de enfermagem para as unidades de internação ${ }^{(10)}$. A utilização estratégica do SCP também instrumentaliza o trabalho de gerenciamento da assistência pelo enfermeiro, logo, favorece sua ação gerencial em prol do cuidado direto ${ }^{(9-12)}$. Apesar disso, estudos que investiguem o dimensionamento em si, relacionando-o à qualidade do cuidado de enfermagem, são incipientes, já que as pesquisas normalmente correlacionam resultados de qualidade e segurança assistencial com a carga de trabalho ${ }^{(5-8)}$ e não com o dimensionamento de pessoal. Neste sentido, acredita-se que pesquisas que comparem a (in)adequação de pessoal de enfermagem com medidas de qualidade assistencial são tanto importantes como necessárias, e podem culminar em inovações de cunho científico (para suscitar métodos mais acurados de investigação) e também em prática de gestão da qualidade e administração de recursos humanos de enfermagem.

Frente às necessidades organizacionais constantemente mutáveis e também à importância de estudos que utilizem o benchmarking na enfermagem como forma de disseminar tal prática de gestão da qualidade dos serviços, emerge a relevância social e científica de investigar mais e de forma conjunta o dimensionamento de pessoal de enfermagem e a qualidade do cuidado.

Ante o exposto, emergiu o impulso de responder a seguinte indagação: Existe diferença na qualidade da assistência expressa por indicadores e no dimensionamento da equipe de enfermagem entre unidades de internação hospitalar? Para tanto, o objetivo deste estudo consistiu em realizar benchmarking interno de indicadores de qualidade e do dimensionamento de pessoal de enfermagem entre unidades de internação hospitalar.

\section{Método}

Trata-se de um estudo transversal, descritivo e analítico, norteado pela metodologia de comparação (benchmarking) interna ${ }^{(2)}$ entre unidades de internação hospitalar. A pesquisa foi desenvolvida em um hospital universitário público do Centro-Oeste do Brasil, que contava com 124 leitos exclusivamente vinculados ao Sistema 
Único de Saúde (SUS). Os setores propriamente pesquisados foram as unidades de internação em clínica médica e cirúrgica, que possuíam 30 e 24 leitos, respectivamente. As unidades foram escolhidas pela internação mútua não crítica de adultos no hospital de inquérito, o que foi entendido como viabilidade para o benchmarking pretendido.

A população da pesquisa foi constituída por pacientes internados nas unidades referidas, no período de maio a julho de 2019. Não houve cálculo amostral, uma vez que a intenção foi alcançar um censo da população elegível no recorte temporal estabelecido.

A amostra foi definida em consonância com os seguintes critérios de inclusão: pacientes com mais de 18 anos de idade; com orientação preservada no tempo e no espaço ou na presença de acompanhante para aqueles com condições clínicas desfavoráveis à resposta e orientação no tempo e no espaço. Pacientes menores de 18 anos de idade, inconscientes e/ou sem acompanhante foram excluídos.

Os dados foram coletados em 20 dias aleatórios durante o período definido, por meio da observação do ambiente de internação dos pacientes, com base em instrumento ${ }^{(13)}$ validado para levantamento de indicadores de qualidade e pela classificação dos pacientes. Foram extraídas, por observação direta, as informações para a definição e comparação de nove indicadores, a saber: identificação do paciente (pelo leito); identificação do paciente (por pulseira); identificação de acessos venosos periféricos; identificação de equipos; identificação de frascos de soro; identificação de sonda nasoenteral (SNE) e/ou sonda nasogástrica (SNG); fixação da sonda vesical de demora (SVD); posicionamento da bolsa coletora de SVD; e posicionamento do prolongamento de drenagem distal da bolsa coletora de SVD ${ }^{(13)}$.

Além da observação para o levantamento dos indicadores, para viabilizar o dimensionamento de pessoal de enfermagem, foi aplicado aos mesmos pacientes observados um Sistema de Classificação de Pacientes (SCP), o qual permite classificar o cliente quanto ao grau de dependência em relação à equipe de enfermagem ou nível de complexidade assistencial ${ }^{(12)}$. O SCP em questão avalia o paciente nas seguintes áreas de cuidado: estado mental, oxigenação, sinais vitais, motilidade, deambulação, alimentação, cuidado corporal, eliminação, terapêutica, integridade cutâneo-mucosa/comprometimento tecidual, curativos e tempo utilizado na realização de curativos. Com base nessa avaliação - que gera uma pontuação com intervalo de 12 a 34 pontos -, os pacientes são classificados em uma das seguintes categorias: cuidados mínimos, cuidados intermediários, cuidados de alta dependência, cuidados semi-intensivos ou cuidados intensivos ${ }^{(12)}$.

Como já era de conhecimento prévio que, na unidade de clínica cirúrgica, em muitos momentos, existem pacientes que não estão no leito, pois está sendo realizado o próprio procedimento cirúrgico, exames ou mesmo esses indivíduos estão deambulando no hospital, o número de pacientes/sítios de observação $(n=57)$ que não estava na unidade no momento da coleta de dados, mas estava internado no setor, foi ajustado proporcionalmente às classificações realizadas pelo SCP, a fim de não interferir (subestimar) no dimensionamento de pessoal de enfermagem da unidade. Isso foi decidido porque a aplicação do SCP na unidade cirúrgica ainda não estava consolidada à época do estudo e, portanto, poderia inviabilizar a recuperação de dados documentais posteriores.

A coleta dos dados referentes aos indicadores de qualidade e classificação de pacientes foi procedida manualmente por duas acadêmicas de enfermagem do último semestre do curso de graduação. Estas foram previamente treinadas por docente, enfermeiro, doutor e pesquisador da área de gerenciamento em enfermagem, além de mestranda em enfermagem e outra docente de enfermagem clínica, mestre, que acompanharam teste piloto e discussão a respeito de possíveis dúvidas (com finalidade de padronização) sobre os instrumentos. Após isso, os dados foram tabulados em planilhas eletrônicas do software Microsoft Office Excel ${ }^{\circledR}$.

A análise dos dados foi realizada por meio da estatística descritiva dos indicadores segundo o percentual (\%) de conformidade, isso é, 
adequação de cada indicador aos padrões previamente estabelecidos para seu cumprimento de qualidade, que são baseados em boas práticas ${ }^{(13)}$. Na análise inferencial de comparação dos indicadores entre as unidades de internação, foi utilizado o teste qui-quadrado com correção de Yates, por meio do software $\mathrm{R}^{\circledR}$, versão 3.5.3 e intervalo de confiança de $95 \%$ das proporções. $\mathrm{Na}$ análise inferencial, o nível de significância aceito foi o de 5\%, expresso em p-valor $\leq 0,05$.

Os indicadores foram interpretados e comparados entre as unidades de acordo com a qualidade da assistência, ilustrada por meio de uma classificação da qualidade do cuidado de acordo com a conformidade do item/indicador em avaliação, a saber: assistência segura (100\%), desejável (99-90\%), adequada (89-80\%), limítrofe (79-70\%) e insuficiente (menor que 70\%) ${ }^{(13)}$.

$\mathrm{Na}$ análise do dimensionamento de pessoal de enfermagem, foi aplicada equação própria do COFEN, segundo normativa atual ${ }^{(10)}$, tal qual:

\section{$\mathrm{QP}=\mathrm{THE} \mathrm{X} \mathrm{KM}$}

Onde, QP = Quadro de pessoal (pessoal de enfermagem dimensionado); THE $=$ Total de Horas de Enfermagem; e KM = Constante de Marinho. A KM é um coeficiente deduzido em relação à: jornada de trabalho semanal, Índice de Segurança Técnica (IST) e dias da semana trabalhados, sendo sete no ambiente hospitalar ${ }^{(10)}$. Destaca-se que o IST representa um percentual adicional ao quadro de pessoal para suprir as ausências previstas e não previstas da equipe de enfermagem, que deve ser de, no mínimo, $15 \%{ }^{(10)}$.

A KM de escolha de cada unidade de internação foi aquela de referência à jornada de trabalho semanal mais prevalente na equipe, em cada setor, assumindo-se IST de 15\%. Assim, a KM utilizada no dimensionamento da unidade de clínica médica foi de 0,2236 (jornada de trabalho semanal prevalente de $36 \mathrm{~h}$ ); e no setor de internação cirúrgica, foi de 0,2012 , pois a jornada de trabalho semanal prevalente entre os membros da equipe de enfermagem era de $40 \mathrm{~h}$ semanais $^{(10)}$.

Para o cálculo de THE, utilizou-se os parâmetros de horas de enfermagem/dia relacionados a cada categoria de classificação do SCP e dispostos na normativa brasileira vigente ${ }^{(10)}$, os quais foram multiplicados pelas médias diárias de pacientes de cada uma dessas categorias do SCP, de acordo com o período de coleta de dados, e por unidade de internação. Já a proporção de enfermeiros e trabalhadores de nível médio no quadro de pessoal dimensionado levou em conta a categoria de cuidado do SCP que demandou maior carga de trabalho (horas de enfermagem requeridas) em cada unidade ${ }^{(10)}$.

Para verificar o número de profissionais disponíveis nos setores (dito quadro de pessoal real), foram consultadas as escalas de trabalho do mês de julho de 2019, que faziam parte do período de referência da coleta de dados. Este número, que era de 41 profissionais (8 enfermeiros e 33 técnicos/auxiliares de enfermagem) na clínica médica e 30 ( 7 enfermeiros e 23 técnicos/auxiliares de enfermagem) na clínica cirúrgica, foi comparado aos respectivos quadros de pessoal dimensionados.

O estudo respeitou integralmente as recomendações éticas da Resolução no 466/2012 do Conselho Nacional de Saúde. A pesquisa integra um Projeto Matricial intitulado "Dimensionamento de Pessoal de Enfermagem, Indicadores de Qualidade e Segurança no Ambiente Hospitalar", o qual foi submetido e aprovado por Comitê de Ética em Pesquisa institucionalizado, recebendo parecer favorável com protocolo de no 3.181.185/2019 e Certificado de Apresentação de ApreciaçãoÉtica(CAAE) 07626019.5.0000.5541.

\section{Resultados}

A respeito dos indicadores de qualidade, foram observados, respectivamente nas unidades de Clínica Médica e Clínica Cirúrgica, os seguintes valores totais de sítios de observação, por indicador: identificação do paciente no leito (450 e 274), identificação do paciente por pulseira ( 450 e 274), identificação de acesso venoso (285 e 164), identificação de equipo (201 e 119), identificação de frascos de soro (201 e 119), identificação de SNE e SNG (50 e 22), fixação de SVD (62 e 52), posicionamento bolsa coletora 
6

Benchmarking de indicadores de qualidade e dimensionamento de pessoal de enfermagem entre unidades hospitalares

de SVD (62 e 52), e posicionamento do prolongamento de drenagem distal da bolsa coletora de SVD (62 e 52). Estes são os valores totais de base para a apreciação da conformidade de cada um dos indicadores e em cada unidade de internação descritos na sequência.
A Tabela 1 ilustra a conformidade dos indicadores de qualidade (isto é, itens adequados), o intervalo de confiança das proporções e a comparação analítica, por unidades de internação.

Tabela 1 - Conformidade de indicadores de qualidade e intervalo de confiança, por indicador de qualidade e unidade de internação hospitalar, e comparação da conformidade dos indicadores entre unidades. Centro-Oeste, Brasil - 2019

\begin{tabular}{|c|c|c|c|c|c|}
\hline Indicador & $\begin{array}{l}\text { Cínica } \\
\text { Médica } \\
\text { n (\%) }\end{array}$ & IC $95 \%(1)$ & $\begin{array}{l}\text { Clínica } \\
\text { Cirúrgica } \\
\text { n }(\%)\end{array}$ & IC 95\% (1) & $\begin{array}{l}\text { p-valor } \\
\text { (2) }\end{array}$ \\
\hline Identificação do paciente (Leito) & $362(80,4)$ & {$[76,5-84]$} & $260(94,9)$ & {$[91,6-97,2]$} & $<0,0001$ \\
\hline Identificação do paciente (Pulseira) & $392(87,1)$ & {$[83,7-90,1]$} & $224(81,8)$ & {$[76,7-86,1]$} & 0,063 \\
\hline Identificação de acesso venoso & $207(72,6)$ & {$[67,1-77,7]$} & $82(50)$ & {$[42,1-57,9]$} & $<0,0001$ \\
\hline Identificação de equipo & - & - & - & - & - \\
\hline Identificação de frascos de soro & $187(93)$ & {$[88,6-96,1]$} & $118(99,1)$ & {$[95,4-100]$} & 0,025 \\
\hline $\begin{array}{l}\text { Identificação de sonda nasoenteral } \\
\text { e sonda nasogástrica }\end{array}$ & $44(88)$ & {$[75,7-95,5]$} & $22(100)$ & {$[84,6-100]$} & 0,217 \\
\hline $\begin{array}{l}\text { Fixação de sonda vesical de } \\
\text { demora }\end{array}$ & $32(51,6)$ & {$[38,6-64,5]$} & $16(30,8)$ & {$[18,7-45,1]$} & 0,039 \\
\hline $\begin{array}{l}\text { Posicionamento bolsa coletora de } \\
\text { sonda vesical de demora }\end{array}$ & $62(100)$ & {$[94,2-100]$} & $52(100)$ & {$[93,2-100]$} & - \\
\hline $\begin{array}{l}\text { Posicionamento do prolongamento } \\
\text { de drenagem distal da bolsa coletora } \\
\text { de sonda vesical de demora }\end{array}$ & 62 (100) & [94,2-100] & $51(98,1)$ & {$[89,7-100]$} & 0,092 \\
\hline
\end{tabular}

Fonte: Elaboração própria.

Notas: Sinal convencional utilizado:

- dado numérico igual a zero não resultante de arredondamento.

(1) Intervalo de Confiança de $95 \%$ de proporções.

(2) Teste qui-quadrado com correlação de Yates.

O Quadro 1 sintetiza a classificação da qualidade da assistência entre os indicadores, de acordo com suas conformidades. Com isso, demonstra a comparação/benchmarking por unidade de internação.

Quadro 1 - Benchmarking interno de indicadores de qualidade da assistência de enfermagem, por indicador e unidade de internação

(continua)

\begin{tabular}{|l|l|l|l|}
\hline Indicador & \multicolumn{1}{|c|}{$\begin{array}{c}\text { Qualidade da } \\
\text { Assistência } \\
\text { Clínica Médica }\end{array}$} & $\begin{array}{c}\text { Qualidade da } \\
\text { Assistência } \\
\text { Clínica Cirúrgica }\end{array}$ & $\begin{array}{c}\text { Melhor Unidade / } \\
\text { Equivalência }\end{array}$ \\
\hline Identificação do Paciente (Leito) & Adequada & Desejável & Clínica Cirúrgica \\
\hline $\begin{array}{l}\text { Identificação do Paciente } \\
\text { (Pulseira) }\end{array}$ & Adequada & Adequada & Equivalente \\
\hline Identificação de acesso venoso & Limítrofe & Insuficiente & Clínica Médica \\
\hline Identificação de Equipo & Insuficiente & Insuficiente & Equivalente \\
\hline Identificação de frascos de soro & Desejável & Desejável & Equivalente
\end{tabular}


Quadro 1 - Benchmarking interno de indicadores de qualidade da assistência de enfermagem, por indicador e unidade de internação

\begin{tabular}{|l|l|l|l|}
\hline Indicador & \multicolumn{1}{|c|}{$\begin{array}{c}\text { Qualidade da } \\
\text { Assistência } \\
\text { Clínica Médica }\end{array}$} & $\begin{array}{c}\text { Qualidade da } \\
\text { Assistência } \\
\text { Clínica Cirúrgica }\end{array}$ & $\begin{array}{c}\text { Melhor Unidade / } \\
\text { Equivalência }\end{array}$ \\
\hline $\begin{array}{l}\text { Identificação de sonda } \\
\text { nasoenteral e sonda nasogástrica }\end{array}$ & Adequada & Segura & Clínica Cirúrgica \\
\hline $\begin{array}{l}\text { Fixação de sonda vesical de } \\
\text { demora }\end{array}$ & Insuficiente & Insuficiente & Equivalente \\
\hline $\begin{array}{l}\text { Posicionamento Bolsa coletora de } \\
\text { sonda vesical de demora }\end{array}$ & Segura & Segura & Equivalente \\
\hline $\begin{array}{l}\text { Posicionamento Distal sonda } \\
\text { vesical de demora }\end{array}$ & Segura & Desejável & Clínica Médica \\
\hline
\end{tabular}

Fonte: Elaboração própria.

Por sua vez, a Tabela 2 ilustra os resultados atinentes à classificação da complexidade assistencial, ou nível de dependência dos cuidados de enfermagem, por unidade de internação hospitalar.

Tabela 2 - Número de classificações e média diária, por unidades de internação e nível de complexidade assistencial. Centro-Oeste, Brasil - 2019

\begin{tabular}{l|c|c|c|c|c}
\hline Unidade & $\begin{array}{c}\text { Cuidado } \\
\text { mínimo } \\
\text { (Média) }\end{array}$ & $\begin{array}{c}\text { Cuidado } \\
\text { intermediário } \\
\text { (Média) }\end{array}$ & $\begin{array}{c}\text { Cuidado de Alta } \\
\text { dependência } \\
\text { (Média) }\end{array}$ & $\begin{array}{c}\text { Cuidado } \\
\text { Semi- } \\
\text {-Intensivo } \\
\text { (Média) }\end{array}$ & $\begin{array}{c}\text { Cuidado } \\
\text { Intensivo } \\
\text { (Média) }\end{array}$ \\
\hline Clínica Médica & 198 & 95 & 73 & 38 & 46 \\
Clínica Cirúrgica & $(9,9)$ & $(4,75)$ & $(3,65)$ & $(1,9)$ & $(2,3)$ \\
& 195 & 80 & 46 & 5 & 5 \\
\hline
\end{tabular}

Fonte: Elaboração própria.

A média de pacientes de cada categoria/nível de dependência de cuidados viabilizou a mensuração das horas de enfermagem requeridas diariamente nos setores, conforme ilustra a Tabela 3 .

Tabela 3 - Horas de enfermagem requeridas diariamente, por nível de complexidade assistencial e Total de Horas de Enfermagem, por unidade de internação hospitalar. Centro-Oeste, Brasil - 2019

\begin{tabular}{l|c|c|c|c|c|c}
\hline Unidade & $\begin{array}{c}\text { Horas de } \\
\text { Cuidado } \\
\text { Mínimo }\end{array}$ & $\begin{array}{c}\text { Horas de } \\
\text { Cuidado } \\
\text { Intermediário }\end{array}$ & $\begin{array}{c}\text { Horas de } \\
\text { Cuidado de } \\
\text { Alta } \\
\text { Dependência }\end{array}$ & $\begin{array}{c}\text { Horas de } \\
\text { Cuidado } \\
\text { Semi- } \\
\text {-Intensivo }\end{array}$ & $\begin{array}{c}\text { Horas de } \\
\text { Cuidado } \\
\text { Intensivo }\end{array}$ & $\begin{array}{c}\text { Total de } \\
\text { Horas de } \\
\text { Enfermagem }\end{array}$ \\
\hline Clínica Médica & 39,6 & 28,5 & 36,5 & 19 & 41,4 & 165 \\
Clínica Cirúrgica & 39 & 24 & 23 & 2,5 & 4,5 & 93 \\
\hline
\end{tabular}

Fonte: Elaboração própria.

O Total de Horas de Enfermagem requerido em cada unidade, ao ser equacionado juntamente com a KM de referência de cada setor, determinou os quadros de pessoal dimensionados das equipes de enfermagem das unidades, os quais foram comparados aos quadros reais (disponíveis em escala de trabalho), expresso na Tabela 4. 
Tabela 4 - Benchmarking de quadros de pessoal real e dimensionado, por unidade de internação hospitalar. Centro-Oeste, Brasil - 2019

\begin{tabular}{l|c|c|c|c|c|c|c|c}
\hline $\begin{array}{l}\text { Dimensionamento } \\
\text { de Pessoal de } \\
\text { Enfermagem }\end{array}$ & \multicolumn{3}{c|}{ Quadro Real } & \multicolumn{2}{c}{ Quadro Dimensionado } & \multicolumn{2}{c}{ Déficit / Superávit } \\
\hline $\begin{array}{l}\text { Unidade de } \\
\text { Internação }\end{array}$ & Enfermeiros & $\begin{array}{c}\text { Técnicos/ } \\
\text { auxiliares } \\
\text { enfermagem }\end{array}$ & Total & Enfermeiros & $\begin{array}{c}\text { Técnicos/ } \\
\text { auxiliares } \\
\text { enfermagem }\end{array}$ & Total & Enfermeiros \\
Clínica Médica & 8 & 33 & 41 & 19 & 18 & 37 & -11 \\
elécnicermagem \\
Clínica Cirúrgica
\end{tabular}

Fonte: Elaboração própria.

\section{Discussão}

Os resultados dos indicadores ilustraram qualidade variada, uma vez que houve sua classificação tanto em aspecto satisfatório (identificação do paciente, posicionamento da bolsa coletora de SVD e posicionamento distal SVD), quanto insatisfatórios a respeito da identificação de equipo e fixação de SVD. A diferença na qualidade assistencial entre as unidades foi mais evidente (com significância estatística) nos indicadores identificação do paciente no leito e identificação de acessos venosos, com resultados melhores para a unidade de clínica cirúrgica e clínica médica, respectivamente.

Há ampla variedade da qualidade assistencial e certa equivalência entre os setores. Em outras palavras, a comparação pelo benchmarking interno não apontou nitidamente a "melhor" qualidade de uma ou outra unidade. Este resultado reforça que as práticas de gestão da qualidade não podem ser pontuais, mas, sim, constantes e sistematizadas ${ }^{(1)}$, a fim de corrigir falhas específicas de cada unidade e/ou métrica avaliada e cumprir seu papel de viabilizar a melhoria contínua $^{(3)}$, sem reforçar a cultura punitiva. Acredita-se que essa assertiva fortalece a importância do benchmarking interno realizado, pois, ainda que tenha sido constatada equivalência geral na qualidade dos setores, aspectos pontuais foram observados, o que torna o planejamento e a execução de melhorias mais factíveis.

A comparação de resultados de indicadores é a premissa básica do processo de benchmarking e algo mais palatável às instituições que adotam modelos/sistemas de gestão da qualidade robustos, a exemplo da Acreditação Hospitalar, que pode gerar uma certificação externa da qualidade dos serviços ${ }^{(1)}$. Em contraponto, um estudo com o objetivo de verificar a associação dos indicadores assistenciais e o nível de certificação das instituições concluiu que a instituição com maior nível de certificação não obteve resultados melhores entre os indicadores assistenciais avaliados na pesquisa. Todavia, a organização com maior nível do selo de Acreditação apresentou importante constância em seus resultados, o que foi interpretado pelas autoras como uma cultura de qualidade mais fortalecida ${ }^{(14)}$.

Chama a atenção o fato de a identificação de equipos não ser uma prática adotada pelos setores em sua rotina, sendo classificada como de qualidade insuficiente em ambas as unidades. Nesse sentido, estudo aponta que a identificação inadequada dos dispositivos de infusão venosa pode favorecer a expiração de seus respectivos períodos de validade, gerando inconformidade de qualidade e expondo o paciente a um potencial risco de complicações ${ }^{(15)}$. Sabe-se, ainda, pela prática clínica, que o hospital em questão possui um bundle interno para prevenção de infecção primária de corrente sanguínea, o qual aborda práticas e cuidados que devem ser monitorados, entre eles a troca de sistema de infusão no tempo adequado e sua identificação correspondente.

A maior parte dos frascos de soro que estavam em uso pelos pacientes apresentou-se em conformidade, sendo classificada como uma qualidade de assistência desejável em ambas as unidades de internação. Isso denota que, na instituição, os profissionais da equipe de enfermagem tendem 
a priorizar a identificação de alguns dispositivos assistenciais em detrimento de outros, o que faz emergir a questão da padronização de processos assistenciais como um meio de buscar melhorias para tais divergências ${ }^{(1)}$.

É fundamental que a equipe de enfermagem incorpore saberes científicos e conhecimentos práticos acerca do manejo adequado dos cateteres vasculares e seus dispositivos, já que estes constituem-se na via de administração mais utilizada no cuidado a pacientes hospitalizados; se forem manejados de maneira inadequada, podem constituir-se em um risco para a segurança do paciente ${ }^{(15-16)}$.

A Agência Nacional de Vigilância Sanitária (ANVISA) ${ }^{(16)}$ orientou recentemente a conduta clínica para a manutenção e troca dos equipos e dispositivos complementares, indicando que a troca desses materiais deve ser realizada sempre: nas trocas dos cateteres venosos (periférico ou centrais); a cada 24 horas, quando os equipos forem em administração de infusões intermitentes; e se de infusão contínua, idealmente não devem ser trocados em intervalos inferiores a 96 horas, no intuito de minimizar a manipulação e consequente risco de contaminação. Além disso, a necessidade de substituição dos equipos e dispositivos complementares poderá ser revista, a depender do tipo de solução utilizada, sob suspeita de contaminação ou quando a integridade do produto ou do sistema estiver comprometida ${ }^{(16)}$.

Estudo ${ }^{(15)}$ realizado em um hospital filantrópico do interior do Paraná também observou a identificação dos equipos de soro, encontrando que $35,85 \%$ destes estavam com identificação adequada e 39,39\% não apresentavam identificação. Portanto, aproximadamente $40 \%$ dos pacientes estavam em uso de dispositivos que não atendiam aos critérios de qualidade e segurança ${ }^{(15)}$. Isto posto, retoma-se o protagonismo da equipe de enfermagem na manutenção/cuidados com esses dispositivos de forma padronizada, pois práticas inadequadas podem expor o paciente ao risco de infecções e reações adversas, além de aumentar os custos e o tempo de internação ${ }^{(16)}$.
Postula-se que a padronização de condutas/ processos, no âmbito do cuidado de enfermagem, não é uma garantia legítima da qualidade assistencial, mas, em termos gerenciais, é um norte importante para a verificação sistemática de inconformidades que podem ser melhoradas no cotidiano atribulado de trabalho.

Reforça o pressuposto anterior um estudo recente $^{(17)}$ desenvolvido no interior do estado de São Paulo, o qual apontou que a opinião de 247 trabalhadores de enfermagem refere que a utilização dos Procedimentos Operacionais Padrão (POP) possibilita a prestação de assistência padronizada e em conformidade com parâmetros técnico-científicos respaldados. Contudo, na mesma pesquisa, foi identificado que a deficiência de recursos humanos seria a principal barreira à implantação dessas ferramentas de padronização ${ }^{(17)}$, o que, de certa forma, guarda alguma relação com este estudo, especialmente na unidade de clínica médica, em que a deficiência de enfermeiros (idealmente o gerente do cuidado e, portanto, um possível ator mais aderente às práticas padronizadas) foi grande. Apesar disso, é prudente admitir que as equipes, no quadro geral, não apresentaram déficit de pessoal, ao menos por meio do método de dimensionamento empregado.

No que tange às observações em relação aos acessos venosos periféricos (AVP), estes apresentaram resultado de qualidade limítrofe na clínica médica e insuficiente na clínica cirúrgica. Visando a melhor qualidade de atendimento ao cliente, recomenda-se que, em adultos, o cateter periférico não deve ser trocado em um período menor que 96 horas, isso quando estiver em condições apropriadas, entre elas: sítio de inserção adequado e constantemente avaliado em relação a sinais flogísticos; integridade da pele e do vaso sanguíneo; tipo e duração de terapia venosa em curso prescrita; além da integridade, permeabilidade do dispositivo e manutenção adequada da cobertura $^{(16)}$.

Sobre o indicador "fixação de sonda vesical de demora", observa-se um padrão de qualidade inadequado no atendimento entre as duas unidades, porém, no que se refere ao posicionamento 
prolongado e distal, classificam-se como seguros (adequados). Assim, a não conformidade para fixação do cateter da sonda, classificada como insuficiente, constitui-se como risco de evento adverso, tendo relação estreita com a chance de tração do cateter vesical, podendo ocasionar traumas na uretra durante a mobilização do paciente, além de migração ascendente de patógenos ao haver a movimentação do cateter para a bexiga, situações que aumentam significativamente o risco de infecção do trato urinário ${ }^{(18)}$.

Em relação aos indicadores de identificação do paciente, tanto pelo leito como por pulseira, foram constatados potenciais de melhoria. A finalidade da identificação correta do paciente é reduzir a ocorrência de incidentes, assegurando que o cuidado seja prestado à pessoa para a qual se destina ${ }^{(19-20)}$. Por meio do uso de identificadores (atualmente, recomendado preferencialmente por meio de pulseira), minimizam-se os riscos de erros no atendimento. As pulseiras devem ser de cor branca, com identificação de, no mínimo, dois identificadores, como nome completo, nome da mãe, data de nascimento e número do atendimento ${ }^{(20)}$.

Embora se tenha verificado resultado estatisticamente significativo para o indicador de identificação do paciente no leito, com melhor conformidade na clínica cirúrgica, a clínica médica aderiu mais à identificação por meio de pulseira, que é considerada uma prática mais segura ${ }^{(20)}$. Reforça-se que o monitoramento contínuo da adesão à identificação do paciente coaduna-se com os incrementos na segurança do cuidado. Neste estudo, parece ser um indicador que ainda necessita de consolidação nas duas unidades, apesar de ambas terem apresentado resultados bem melhores se comparadas à internação médico-cirúrgica de um hospital universitário público do Sul do Brasil $(63,9 \%)^{(19)}$.

Seguindo os parâmetros da Resolução COFEN $\mathrm{n}^{\mathrm{o}} 543 / 2017^{(10)}$, seriam necessários 19 enfermeiros no quadro de pessoal da clínica médica, para suprir as necessidades da demanda assistencial. O quadro real de enfermeiros apresentou um déficit em torno de $60 \%$ sobre o quadro projetado, o que pode implicar na sobrecarga de trabalho na categoria e/ou desvio de suas funções sobre o cuidado direto ao paciente, dada à sua maior complexidade/nível de dependência. A sobrecarga, por sua vez, revela-se um importante agravo na saúde profissional de enfermeiros, como estresse e exaustão. Um dos agravos mais encontrados em estudo foi o estresse ocupacional, o que pode implicar negativamente na qualidade do cuidado na saúde do trabalhador ${ }^{(21)}$.

Uma investigação em 11 Unidades de Terapia Intensiva da capital de São Paulo, com o objetivo de verificar a correlação entre tempo de assistência de enfermagem e indicadores de qualidade assistencial, não encontrou correlações significativas, ainda que os autores tenham feito alusões pertinentes a respeito da interrelação entre essas variáveis ${ }^{(22)}$. Somado a isso, apesar de a correlação entre os indicadores e o dimensionamento não ter sido objeto deste estudo e, sim, o benchmarking comparativo, em termos descritivos, parece que não houve diferença na qualidade do cuidado em relação ao quantitativo de pessoal projetado/real. Isso porque, a totalidade da qualidade assistencial pode ser interpretada como equânime entre as unidades. Já sobre a comparação do dimensionamento das equipes e os respectivos quadros reais, ficou evidente o déficit de pessoal de nível superior na clínica médica em comparação à clínica cirúrgica, que, antagonicamente, apresentou maior superávit de pessoal.

Também é prudente e necessário mencionar que, mesmo que a unidade de clínica cirúrgica tenha apresentado maior superávit de pessoal e não tenha apresentado déficit evidente, como na clínica médica, nesse setor, os trabalhadores atuavam - em maior parcela - por 40 horas semanais, o que, naturalmente, demanda menor quantitativo de pessoal, comparando-se à jornada de 36 horas do setor de clínica médica. Acerca disso, é necessário admitir que o dimensionamento na clínica médica estava desajustado à lógica exclusiva dos parâmetros vigentes do COFEN. No entanto, a carga de trabalho mensurada, por exemplo, pelo volume de pacientes atribuídos por trabalhador, conforme já utilizado 
como métrica importante para ser correlacionada com indicadores de qualidade e segurança em outro estudo nacional $^{(5)}$, é fator não considerado neste estudo, que optou por seguir uma lógica padronizada de avaliação, que foi eminentemente comparativa em termos de paralelo entre duas unidades. Isto é, outras medidas de carga de trabalho para além do SCP e da jornada de trabalho semanal (culminando com dimensionamento em si) poderiam elucidar mais e melhor a correlação da adequação de recursos humanos de enfermagem com a qualidade assistencial nos setores, o que fica como sugestão para pesquisas futuras.

Há dificuldade de se estabelecer relações diretas do quantitativo geral de pessoal de enfermagem projetado/dimensionado sobre medidas de qualidade do cuidado e segurança do paciente. Talvez, por este motivo, alguns estudos recentes utilizam a razão de pacientes por profissional de enfermagem como um parâmetro para ser associado às métricas de interesse, na qual demonstra-se, tanto no Brasil ${ }^{(5)}$ como nos Estados Unidos da América ${ }^{(23)}$, que a redução do volume de pacientes designado aos trabalhadores de enfermagem ou o maior volume de pessoal (staffing) para atender a uma clientela tende a favorecer melhores resultados com sensibilidade de terem sua qualidade verificada.

O elevado déficit (-11) de enfermeiros associado ao superávit $(+15)$ de pessoal de nível médio encontrado no dimensionamento da unidade de clínica médica é um achado já referido $^{(9,11)}$ quando na investigação de unidades de internação, onde o grau de dependência de cuidados é alto, como foi verificado neste setor. Contudo, novamente, retoma-se que a qualidade do cuidado foi equivalente entre as unidades, o que leva à reflexão de que, além da necessidade de revisão do quadro de pessoal, as próprias iniciativas de planejamento e controle da qualidade do cuidado contêm espaços de melhoria, o que por si só é uma contribuição do estudo à prática de gerenciamento em enfermagem com enfoque na qualidade do cuidado.

Os achados expostos, atrelados à literatura científica, remetem à uma necessária reflexão da enfermagem brasileira, a fim de discutir as diretrizes dos órgãos representativos da profissão e a realidade dos serviços hospitalares. Como em outras pesquisas mencionadas, o número total de profissionais de nível médio aproxima-se ou até mesmo supera o preconizado pela normativa vigente do COFEN. No entanto, os déficits concentram-se no número de enfermeiros, o que indica a premência em repensar os processos de trabalho e a inserção maior do enfermeiro no cuidado direto, com o objetivo de qualificar a assistência, especialmente aquela dispensada para clientela de maior nível de complexidade.

Pensar em reduzir o número total da equipe de enfermagem para atender à demanda projetada de enfermeiros, conforme sugere o dimensionamento da unidade de clínica médica em especial, mas também da cirúrgica, certamente é um grande tabu e desafio, tanto na perspectiva de trabalhadores, que poderiam se sentir lesados, como, talvez, para o cuidado ao paciente. Logo, verifica-se que esta realidade complexa cabe às lideranças de enfermagem equacionarem e também viabilizarem boas condições de trabalho para a equipe e, consequentemente, de cuidado de enfermagem.

Para buscar melhores alternativas para a situação exposta, um período prolongado de análise da complexidade assistencial dos pacientes seria necessário. Além disso, seria igualmente indispensável uma visão mais sistêmica sobre a realidade dos trabalhadores, tais como idade, índices de rotatividade e absenteísmo, entre outros, e também aspectos inerentes (e, portanto, peculiares) ao processo de trabalho em cada unidade hospitalar.

No dimensionamento de pessoal, além do grau de dependência de cuidados da clientela, deve-se considerar características do serviço/instituição de saúde e também do serviço de enfermagem $^{(10)}$. De certa forma, isso é um ponto que faz com que os objetos deste estudo - a qualidade do cuidado e o dimensionamento de pessoal -, investigados em conjunto, ganhem ainda mais relevância, pois percebe-se que, mesmo com equipes, a princípio com superávit de pessoal de enfermagem, a qualidade do cuidado 
tem evidentes espaços para avanço/melhoria, o que denota características do serviço de saúde e de enfermagem que ultrapassam a questão do quantitativo de pessoal disponível.

A equivalência na qualidade assistencial vislumbrada na avaliação pelos indicadores, a clara deficiência de enfermeiros na unidade de clínica médica e o superávit de pessoal na clínica cirúrgica, possivelmente, refletem que a adequação de pessoal não é um fator isolado na qualificação do cuidado. Levanta-se a hipótese acerca dos modelos de supervisão - organizacional e assistencial -, da adoção de processos padronizados, da avaliação sistemática e periódica do cuidado e dos recursos correlatos para a sua produção e da própria ética profissional que são fatores com repercussão (positiva ou não) na qualidade assistencial. Todavia, a racionalização da carga de trabalho e a adequação de pessoal em relação à demanda assistencial, certamente, merecem ser continuamente (re)planejados por gestores de enfermagem comprometidos com o cuidado qualificado e com os próprios trabalhadores.

Acredita-se que a maior limitação deste estudo é a ausência de correlação estatística robusta entre a qualidade assistencial e o dimensionamento de pessoal de enfermagem e/ou a carga de trabalho. Apesar disso, a investigação contribui para o avanço do conhecimento em gestão da qualidade em enfermagem e gerenciamento de recursos humanos, além de divulgar (na enfermagem, que é pouco aderente) o benchmarking como estratégia factível e consistente para planejamento de melhoria de processos assistenciais e também de gestão de pessoas.

\section{Conclusão}

Conclui-se que a qualidade assistencial - mediada pelos indicadores analisados - entre os setores de internação foi equânime e o dimensionamento de pessoal foi discrepante, uma vez que a equipe da clínica médica tinha evidente déficit de enfermeiros dada à maior complexidade assistencial atendida. Entretanto, é prudente ressaltar a maior jornada de trabalho semanal na equipe da clínica cirúrgica em comparação com a da clínica médica, o que atribuiu número reduzido na previsão do quadro de pessoal dimensionado nesse setor. Isso reforça que a correlação entre a qualidade do cuidado e o dimensionamento de pessoal de enfermagem merece maior investigação e integração.

Apesar de a pesquisa restringir-se a uma localidade pontual, acredita-se que o estudo contribui para o avanço científico, ao suscitar a necessidade do incremento de métodos que viabilizem paralelos da qualidade assistencial com o provimento de recursos humanos de enfermagem, seja de forma correlacional, ou seja, por meio eminentemente comparativo, como no caso do benchmarking realizado, o que extrapola o interesse local. Sobretudo, o estudo é um possível sinalizador de que a melhoria da qualidade do cuidado é um objetivo complexo que deve levar em conta não "somente" a adequação de pessoal, ainda que este aspecto seja inegavelmente relevante ou, até mesmo, protagonista para o seu alcance.

\section{Colaborações:}

1 - concepção, projeto, análise e interpretação dos dados: João Lucas Campos de Oliveira, Margani Cadore Weis Maia, Thamyres Laiz Oliveira Aquino e Samanta de Cassia da Silva;

2 - redação do artigo e revisão crítica relevante do conteúdo intelectual: João Lucas Campos de Oliveira, Margani Cadore Weis Maia, Ana Maria Müller de Magalhães, Rúbia Marcela Rodrigues Moraes e Michelle Dornelles Santarem; 3 - aprovação final da versão a ser publicada: João Lucas Campos de Oliveira, Margani Cadore Weis Maia, Ana Maria Müller de Magalhães, Rúbia Marcela Rodrigues Moraes, Michelle Dornelles Santarem, Thamyres Laiz Oliveira Aquino e Samanta de Cassia da Silva.

\section{Referências}

1. Oliveira JLC, Gabriel CS, Fertonani HP, Matsuda LM. Management changes resulting from hospital accreditation. Rev latino-am enfermagem. 2017;25:e2851. DOI: http://dx.doi. org/10.1590/1518-8345.1394.2851 
João Lucas Campos de Oliveira, Margani Cadore Weis Maia, Ana Maria Müller de Magalhães, Rúbia Marcela Rodrigues Moraes, Michelle Dornelles Santarem, Thamyres Laiz Oliveira Aquino, Samanta de Cassia da Silva

2. Bitar OJNV. Indicadores de qualidade e quantidade em saúde. RAS [Internet]. 2001 [cited 2020 Jun 18];3(12):21-8. Available from: http://www.saude.am.gov.br/planeja/doc/ indicadores.pdf

3. Tardy AE, Levif M, Michel P. Benchmarking: a method for continuous quality improvement in health. Healthc Policy [Internet]. 2012 [cited 2020 Jun 20];7(4):e101-19. Available from: https://www. ncbi.nlm.nih.gov/pmc/articles/PMC3359088/pdf/ policy-07-e101.pdf

4. Cho E, Sloane DM, Kim E-Y, Kim S, Choi M, Yoo IY, et al. Effects of nurse staffing, work environments, and education on patient mortality: An observational study. Int J Nurs Stud. 2015;52(2):535-42. DOI: 10.1016/ j.ijnurstu.2014.08.006

5. Magalhães AMM, Costa DG, Riboldi CO, Mergen T, Barbosa AS, Moura GMSS. Association between workload of the nursing staff and patient safety outcomes. Rev Esc Enferm USP. 2017;51:e03255. DOI: http://dx.doi.org/10.1590/ S1980-220X2016021203255

6. Fagerström L, Kinnunen M, Saarela J. Nursing workload, patient safety incidents and mortality: an observational study from Finland. BMJ Open. 2018;8:e016367. DOI:10.1136/ bmjopen2017-016367

7. Chang LY, Yu H-H, Chao CY-F. The Relationship Between Nursing Workload, Quality of Care, and Nursing Payment in Intensive Care Units. J Nurs Res. 2019;27(1):1-9. DOI: 10.1097/jnr. 0000000000000265

8. Cuadros KC, Padilha KG, Toffoletto MC, Henriquez-Roldán C, Canales MAJ. Patient Safety Incidents and Nursing Workload. Rev latino-am enfermagem. 2017;25:e2841. DOI: http://dx.doi. org/10.1590/1518-8345.1280.2841

9. Vasconcelos OV, Rogo DFH, Marques LGS, Tonini NS, Oliveira JLC. Dimensioning of hospital nursing personnel: study with brazilian official parameters of 2004 and 2017. Esc Anna Nery Rev Enferm. 2017;21(4):e20170098. DOI: 10.1590/2177-9465-EAN-2017-0098

10. Brasil. Conselho Federal de Enfermagem. Resolução n 543/2017. Atualiza e estabelece parâmetros para o Dimensionamento do Quadro de Profissionais de Enfermagem nos serviços/ locais em que são realizadas atividades de enfermagem [Internet]. Brasília (DF); 2017 [cited
2020 Jun 2019]. Available from: http://www. cofen. gov.br/ resolucao-cofen-5432017_51440.html

11. Pedro DRC, Ribeiro DB, Sorrilha MM, Tonini NS, Haddad MCFL, Oliveira JLC. Dimensioning of nursing in a hospital department of detoxification for drug abuse. Cienc Cuid Saúde. 2018;17(4):e43769. DOI: 10.4025/cienccuidsaude.v17i4.43769

12. Santos F, Rogenski NMB, Baptista CMC, Fugulin FMT. Patient classification system: a proposal to complement the instrument by Fugulin et al. Rev latino-am enferm 2007;15(5):980-5. DOI: http:// dx.doi.org/10.1590/S0104-11692007000500015

13. Vituri DW. Avaliação como princípio da Gestão da Qualidade: testando a confiabilidade Inter avaliadores de indicadores para avaliação da qualidade da assistência de enfermagem (tese). Ribeirão Preto (SP): Universidade de São Paulo; 2013.

14. Braga A, Pena M, Melleiro M. Metrics of assistance indicators of certified hospitals. Rev enferm UFPE online. 2018;12(3):665-75. DOI: https://doi.org/10.5205/1981-8963-v12i3a230715p665675-2018

15. Souza VS, Amorim DO, Silva NB, Stevanato KP, Melo WA, Costa MAR. Indicators of quality of nursing assistance in peripheral intravenous therapy. Rev enferm UFPE online. 2017;11(5):1989-95. DOI: 10.5205/reuol.93020 -81402-1-RV.1105sup201703

16. Agência Nacional de Vigilância Sanitária. Medidas de Prevenção de Infecção Relacionada à Assistência à Saúde [Internet]. Brasília (DF); 2017 [cited 2020 Jun 20]. Available from: http:// portal.anvisa.gov.br/s/33852/3507912/+4++M edidas+de+Preven $\% \mathrm{C} 3 \% \mathrm{~A} 7 \% \mathrm{C} 3 \% \mathrm{~A} 3 \mathrm{O}+\mathrm{de}+\mathrm{Inf}$ ec\%C3\%A7\%C3\%A3o+Relacionada+\%C3\%A0+ Assist\%C3\%AAncia+\%C3\%A0+Sa\%C3\%BAde/ a3f23dfb-2c54-4e64-881c-fccf9220c373

17. Sales CB, Bernardes A, Gabriel CS, Brito MFP, Moura AA, Zanetti ACB. Standard Operational Protocols in professional nursing practice: use, weaknesses and potentialities. Rev bras enferm. 2018;71(1):126-34. DOI: http://dx.doi. org/10.1590/0034-7167-2016-0621

18. Mota EC, Oliveira AC. Catheter-associated urinary tract infection: why do not we control this adverse event? Rev esc enferm USP. 2019;53:e03452. DOI: http://dx.doi.org/10.1590/ s1980-220x2018007503452 
19. Fujii Neta A, Girardi C, Santos DTR, Oliveira JLC, Oliveira RP, Maraschin MS, et al. Adesão à identificação do paciente em hospital universitário público. Rev Adm Saúde. 2018;18(70). DOI: http://dx.doi.org/10.23973/ras.70.70

20. Brasil. Ministério da Saúde. Protocolo de identificação do paciente [Internet]. Brasília (DF); 2013 [cited 2020 Jun 12]. Available from: http:// www20.anvisa.gov.br/segurancadopaciente/ index.php/publicacoes/item/identificacaodopaciente

21. Dalri RCMB. Nurses' workload and its relation with physiological stress reactions. Rev latino-am enfermagem. 2014;22(6):959-65. DOI: $10.1590 / 0104-1169.3292 .2503$
22. Garcia PC, Tronchin DMR, Fugulin FMT. Care time and quality indicators in Intensive Care Units. Rev Bras Enferm. 2019;72(Suppl 1):166-72. DOI: http://dx.doi.org/10.1590/0034-7167-2018-0067

23. Carlisle B, Perera A, Stutzman SE, Brown-Cleere S, Parwaiz A, Olson DM. Efficacy of using available data to examine nurse staffing ratios and quality of care metrics. J Neurosci Nurs. 2020;52(2):78-83. DOI: $10.1097 / \mathrm{JNN} .0000000000000499$

Recebido: 4 de julho de 2020

Aprovado: 13 de agosto de 2020

Publicado: 30 de setembro de 2020

A Revista Baiana de Enfermagem utiliza a Licença Creative Commons - Atribuição-NãoComercial 4.0 Internacional.

https://creativecommons.org/licenses/by-nc/4.0/

Este artigo é de acesso aberto distribuído sob os termos da Licença Creative Commons (CC BY-NC).

Esta licença permite que outros remixem, adaptem e criem a partir do seu trabalho para fins não comerciais. Embora os novos trabalhos tenham de lhe atribuir o devido crédito e não possam ser usados para fins comerciais, os usuários não têm de licenciar esses trabalhos derivados sob os mesmos termos. 